\title{
PLANTAS VASCULARES DE LA BAHÍA DE JULI, LAGO TITICACA, PUNO-PERÚ
}

\section{VASCULAR PLANTS FROM THE BAY OF JULI, TITICACA LAKE, PUNO-PERÚ}

\author{
María Arteta $^{1}$, Massiel Corrales ${ }^{2}$, Carla Dávalos ${ }^{3}$, Amalia Delgado ${ }^{4}$, Felipe Sinca ${ }^{5}$, Lizbeth Hernani ${ }^{6}$ y Javier \\ Bojórquez $^{7}$
}

\section{Resumen}

Se presenta la riqueza específica de las plantas vasculares de la Bahía de Juli en el sector de Huaquina y comunidades de Olla y C’aje-Huaylluni, a orillas del Lago Titicaca, desde los 3830 hasta los 4200 m de altitud, de Marzo a Mayo del 2006. La herborización y determinación de las muestras colectadas se realizó en el Herbarium Arequipense (HUSA) de la Universidad Nacional de San Agustín de Arequipa. Se hallaron 185 especies correspondientes a las divisiones Pteridophyta, Pinophyta y Magnoliophyta, agrupadas en 58 familias y 137 géneros; dentro de las Magnoliophyta las familias Asteraceae y Poaceae son las mejor representadas, las Pteridaceae y las Dryopteridaceae tienen mayores porcentajes dentro de Pteridophyta. Pinophyta solo reportó 3 especies. El sector Huaquina presentó la mayor riqueza, pudiéndose encontrar 136 de estas especies. Cabe resaltar que 47 (25.4\%) de estas especies son nuevos registros para el departamento de Puno.

Palabras clave: Riqueza, plantas vasculares, Juli, Lago Titicaca

\begin{abstract}
The vascular plant species richness is presented for the bay of Juli, specifically the Huaquina sector and the communities of Olla and C'aje-Huaylluni, on the Titicaca Lake shore. This area was studied from March to May 2006, covering altitudinal ranges of 3830 up to 4200 masl. Drying, mounting and determination of the collected samples were performed at the Herbarium Arequipense (HUSA) of the Universidad Nacional de San Agustín de Arequipa. A total of 185 species were found, belonging to the divisions: Pteridophyta, Pinophyta and Magnoliophyta and can be grouped into 58 families and 137 genera; Asteraceae and Poaceae families had most of the species among Magnoliophyta; within Petridophyta, Pteridaceae and Dryopteridaceae presented the highest percentages. Only 3 species were found in Pinophyta. The richest sector was Huaquina, where 136 species could be found. A very striking fact is that $25.4 \%$ (47 spp.) of the species were new records for the Puno department.
\end{abstract}

Key words: Richness, vascular plants, Juli, Titicaca Lake

\section{Introducción}

Pese a que se han realizado algunas investigaciones en el departamento de Puno, no se tienen registros de flora en la provincia de Chucuito y más específicamente en el distrito de Juli, lugar que posee una gran riqueza cultural, histórica y también biológica donde se encuentran especies vegetales bajo alguna categorización de amenaza como Polylepis incana Kunth y Budlleja incana Ruiz \& Pav. Que se encuentran actualmente en peligro crítico (INRENA, 2006).

La flora presente en las comunidades de estudio, es de vital importancia debido a que los pobladores la usan con fines alimenticios, medicinales, energéticos, etc. Actualmente, uno de los principales problemas, es la pérdida de biodiversidad, ocasionada por la reforestación con especies exóticas como Eucalyptus globulus Lab. y Cupressuss sempervirens L., donde la primera es bien conocida por degradar y desecar los suelos (Odum, 1972).
Debido a que Juli se encuentra cercana a la Reserva Nacional del Titicaca y a la Zona Reservada Aymara Lupaca, compartiendo ecosistemas y recursos similares y teniendo potencial para ser incluida dentro del Sistema Nacional de Áreas Naturales Protegidas por el Estado (SINANPE), es que el objetivo del presente trabajo es dar a conocer la diversidad de plantas vasculares de la Bahía de Juli, como primer paso a posteriores estudios y a su posible conservación.

\section{Material y métodos}

Área de estudio

Para el desarrollo de la presente investigación, se tomó como referencia el circuito turístico Huaquina Olla - Huaylluni, donde se ubicaron tres zonas principales de muestreo (Figura 1):

Zona 1: Sector de Huaquina (Isla del Pato), localizada a los $16^{\circ} 12^{\prime} 04,2^{\prime \prime}$ ' Latitud Sur y 69²8'13,3'’ Longitud Oeste, con altitudes entre 3834 y 4200 m. 
Zona 2: Comunidad de Olla (laguna de oxidación), localizada a los $16^{\circ} 12^{\prime} 23^{\prime \prime}$ ' LS y 69'26'36'” LO, con altitudes entre 3861 y $4008 \mathrm{~m}$.

Zona 3: Comunidad de C'aje - sector Huaylluni, localizada a los $16^{\circ} 11^{\prime} 8,9^{\prime \prime}$ ' LS hasta 69²4'59.5', LO, altitudes entre $3830 \mathrm{~m}$ y $3900 \mathrm{~m}$.

Figura 1. Mapa de ubicación de las zonas de muestreo en la Bahía de Juli, sector de Huaquina, comunidades de Olla y C’aje - Huaylluni Provincia de Chucuito, Puno - Perú.

杄 Zonas de muestreo, $\mathrm{C}^{0}$ Cerros

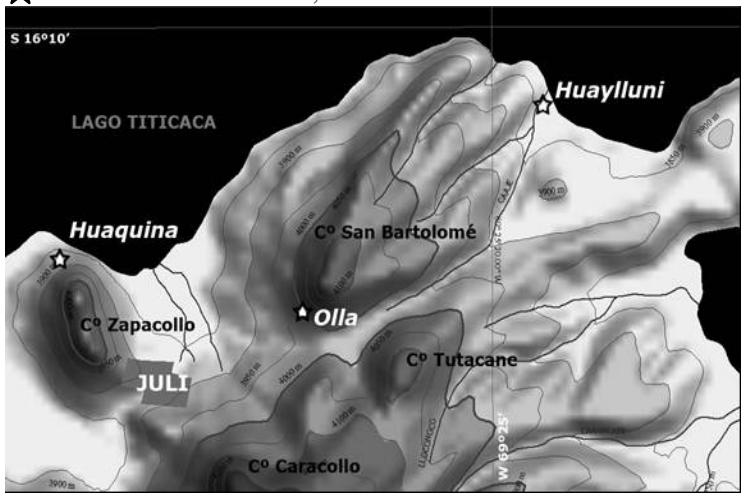

Descripción de la zona de estudio

Clima: El distrito de Juli, se caracteriza por presentar un clima seco y frío, con temperaturas que oscilan entre los $0{ }^{\circ} \mathrm{C}$ y $15{ }^{\circ} \mathrm{C}$.y una estación lluviosa que se inicia en septiembre, extendiéndose hasta los meses de marzo-abril (Valdivia, 2005).

Fisiografía: La zona de estudio está conformada por dos unidades fisiográficas; la circunlacustre, comprendida por áreas ubicadas entre los 3812 y 3880 msnm; presenta suelos con relieves planos, medianamente accidentados y accidentados y la intermedia, que ocupa áreas entre los 3881 y 4600 msnm; con relieves bastante accidentados, propensos a erosión pluvial y eólica, siendo la circunlacustre la predominante, la misma que es vulnerable a los efectos de las precipitaciones pluviales que saturan el suelo y producen deslizamientos de los taludes que tienen pendientes considerables (Valdivia, 2005).

Geología: La zona de estudio posee suelos de baja permeabilidad, formados por depósitos de arcilla plástica, limos y material orgánico de origen pluvioglaciar. También se puede notar la presencia de material conglomerado, formado por la mezcla de materiales granulares y finos de origen pluvial. Asimismo, es frecuente notar la presencia de afloramientos de rocas sedimentarias, especialmente de rocas calizas que se encuentran fracturadas, trituradas y alteradas (Valdivia, 2005).

Ecología: Juli se encuentra en la zona de vida Bosque Húmedo Montano Subtropical (bh-Ms), que a pesar de tener una precipitación no mayor de $800 \mathrm{~mm}$ anuales y una reducida evapotranspiración debido a las bajas temperaturas, permiten llevar a cabo una agricultura de secano. En este sentido, constituyen una zona de vida donde se fija el centro de la agricultura de secano del país, donde se cultiva plantas autóctonas de gran valor alimenticio (Holdridge, 1978).

Vegetación: La vegetación natural ha sido modificada por el hombre observándose árboles esparcidos de Escallonia sp. "chachacomo" y Polylepis sp. "queñual”. Es muy frecuente Lupinus mutabilis Sweet "tarwi" cerca de los caminos (Valdivia, 2005).

Se observa grandes extensiones de pastos naturales altoandinos, constituidos principalmente por especies de la familia Poaceae como Eragrostis, Stipa, Calamagrostis, Festuca y Poa, entre las más importantes (Holdridge, 1978).

Metodología

Se realizaron excursiones al sector de Huaquina, comunidad de Olla y C'aje - Huaylluni, con la finalidad de colectar la mayor cantidad de especies presentes en la zona. El material botánico se colectó según el método clásico u ortodoxo (Schjellerup et al., 2005). Se tomaron datos in situ de la ubicación geográfica, altitud y estado en el que se encontraron los diferentes especimenes. La determinación de las especies se realizó en el Herbarium Arequipense (HUSA) de la Universidad Nacional de San Agustín de Arequipa, con ayuda de especialistas, literatura especializada, y por comparación con muestras presentes en el Herbario.

Para el procesamiento de los datos, las especies fueron ordenadas alfabéticamente por familias dentro de los grupos taxonómicos de mayor jerarquía (divisiones Magnoliophyta, Pinophyta y Pteridophyta); el sistema de clasificación empleado para Magnolipohyta fue el de Cronquist (1981), Pteridophyta el de Tryon (1989) y Pinophyta el de Brako \& Zarucchi (1993).

\section{Resultados y discusión}

Flora vascular:

Se registraron un total de 185 taxa agrupados en 3 divisiones, 58 familias y 137 géneros. Los resultados muestran la gran diversidad florística que existe en esta zona, principalmente en el anillo circunlacustre (Tabla 1). La división Magnoliophyta es la mejor representada con el 84.5\% (170 spp.), le sigue Pteridophyta con el 10.3\% (12 spp.) y por último Pinophyta con 5.2\% (3 spp.). Cabe resaltar que dentro de Magnoliophyta, la clase Magnoliopsida fue la más abundante con 138 especies (Figura 2). Se reportaron 47 nuevos registros de especies para el departamento de Puno, ampliando su rango de distribución (Brako \& Zarucchi, 1993). 
Tabla 1. Flora vascular registrada en el Área de la Bahía de Juli, sector de Huaquina, comunidades de Olla y C’aje-Huaylluni, en el Lago Titicaca, Departamento de Puno, 2006

\begin{tabular}{lcccccc}
\hline \multirow{2}{*}{ DIVISIONES } & \multicolumn{2}{c}{ FAMILIA } & \multicolumn{2}{c}{ GENERO } & \multicolumn{2}{c}{ ESPECIE } \\
\cline { 2 - 7 } & $\mathrm{N}$ & $\%$ & $\mathrm{~N}$ & $\%$ & $\mathrm{~N}$ & $\%$ \\
\hline Magnoliophyta & 49 & 84.5 & 123 & 89.8 & 170 & 91.9 \\
Pinophyta & 3 & 5.2 & 3 & 2.2 & 3 & 1.6 \\
Pteridophyta & 6 & 10.3 & 11 & 8.0 & 12 & 6.5 \\
\hline TOTAL & 58 & & 137 & & 185 & \\
\hline
\end{tabular}

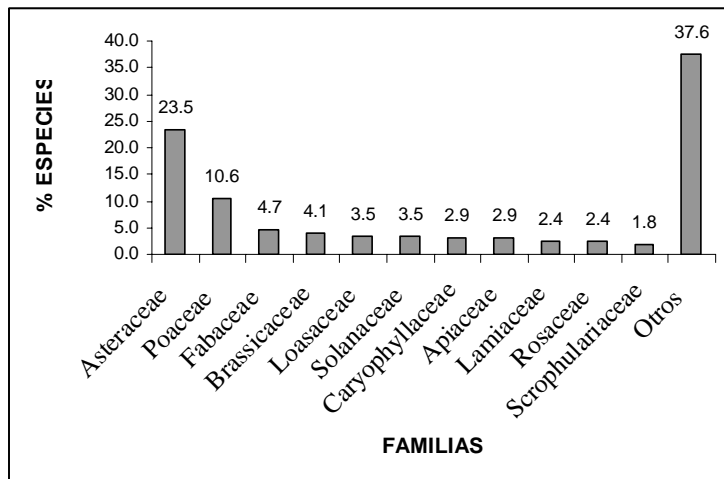

Figura 2. Familias de Magnoliophyta con mayor número de especies en la Bahía de Juli, sector de Huaquina, comunidades de Olla y C'aje - Huaylluni, 2006.

Las familias con mayor riqueza de géneros y especies fueron: Asteraceae con Pseudognaphalium (4 spp.), Hypochaeris (3 spp.), Tagetes, Sonchus, Gamochaeta, Galinsoga, Cotula, Conyza y Bidens (2 spp. cada uno), además de otros 18 géneros con una sola especie; así mismo las Poaceae estuvieron representadas por Eragrostis (3 spp.), Bromus, Hordeum, Poa y Stipa (2 spp. cada uno) y otros 7 géneros con una sola especie (Anexo 1). La familia Asteraceae posee una gran diversidad de especies y géneros en todo el mundo, debido a su gran plasticidad genética, logrando adaptarse a la mayoría de los ambientes y a su fácil dispersión; este comportamiento es compartido con las Poaceae (Schjellerup et al., 2005). Esto concuerda con lo reportado por Brako \& Zarucchi (1993), que indican que en zonas altoandinas ambas familias son las que registran mayor diversidad.

La división Pteridophyta estuvo representada por 6 familias (Anexo 2), siendo las Pteridaceae, las que presentaron la mayor cantidad de géneros: Pellaea, Argyrochosma, Cheylanthes y Eriosorus, seguidas por las Dryopteridaceae: Cystopteris, Polystichum y Woodsia, ambas representan el $66.6 \%$ del total de pteridofitas (Figura 3). La mayoría de las especies se encontraron en lugares húmedos y en grietas de rocas que brindan condiciones favorables para el desarrollo de los diferentes géneros encontrados (Cystopteris, Asplenium, Adiantum, Equisetum y Woodsia); también se encontraron especies xerofíticas en zonas llanas (Argyrochosma, Pellaea, Polystichum y Cheylanthes) y una sola especie acuática Azolla filiculoides Lam.

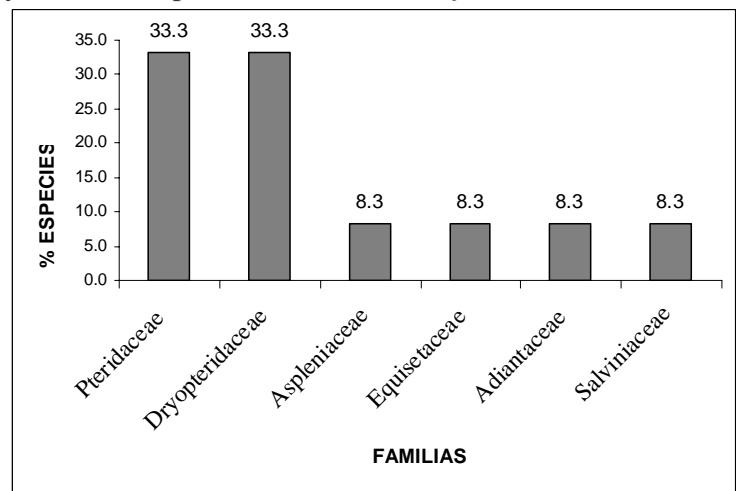

Figura 3. Familias de Pteridophyta con mayor número de Especies en Bahía de Juli, sector de Huaquina, comunidades de Olla y C’aje - Huaylluni, 2006.

León (1996) reportó 213 especies de Pteridofitos para el departamento de Puno, considerándolo como uno de los departamentos con mayor diversidad en el Perú. Asimismo, menciona que en la vertiente occidental las familias más diversas en especies son Pteridaceae, Polypodiaceae y Dryopteridaceae. León \& Young, (1996) señalan la existencia de 29 especies de Pteridofitos en la cuenca del Titicaca.

La división Pinophyta presentó 3 especies, de las cuales Pinus radiata L. y Cupressus sempervirens L., son especies introducidas que han venido siendo utilizadas con fines de reforestación.

El $73.5 \%$ de las plantas vasculares registradas, se encontraron en el sector de Huaquina, seguidas por la comunidad de C'aje - Huaylluni con 59.5\% y la comunidad de Olla con 54.6\%. La mayoria de las especies registradas se repiten en las zonas de muestreo (Figura 4). El 4.3\% del total registrado correponde a las especies acuáticas y semiacuaticas; sin embargo el INRENA (2002) reportó 17 especies de plantas acuáticas y semiacuaticas, en un inventario para toda la Reserva Nacional del Titicaca.

Especies como Azolla filiculoides Lam. y Lemna minuta Kunth sólo se registraron en la Comunidad de Olla y al estar presentes cerca de la laguna de Oxidación, indicarían la ocurrencia de procesos de eutroficación.

\section{Conclusiones}

En la Bahía de Juli, Sector de Huaquina y Comunidades de Olla y C`aje-Huaylluni, se registraron 185 taxa agrupados en 3 divisiones, 58 familias y 137 géneros. Las familias con mayor riqueza de géneros y especies fueron Asteraceae (27 géneros y 40 especies) y Poaceae (12 géneros y 18 especies).

Las división Pteridophyta estuvo representada por 6 familias: Adiantaceae, Aspleniaceae, 
Dryopteridaceae, Equisetaceae, Pteridaceae y Salviniaceae.

La división Pinophyta, solo registro tres especies: Pinus radiata L., Cupresus sempervirens L. y Ephedra americana Humb. \& Bonpl. ex Willd.

Se reportan 47 nuevos registros para el departamento de Puno, ampliando su rango de distribución.

El sector de Huaquina fue el que presentó mayor diversidad de especies (136).

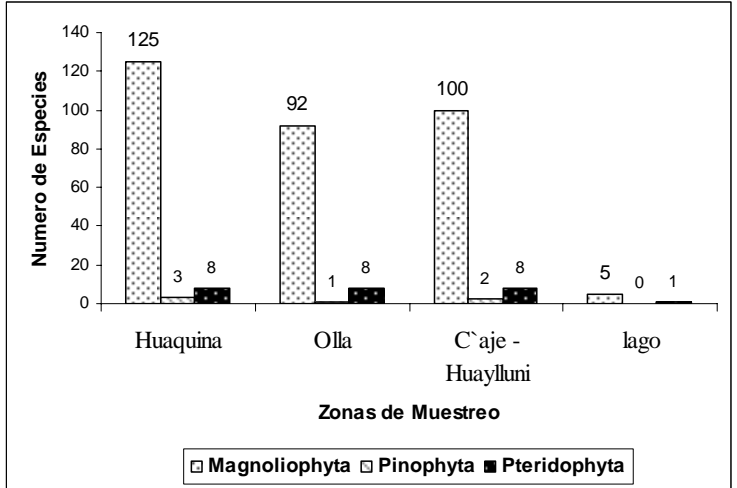

Figura 4. Plantas vasculares con mayor número de especies por zona de muestreo en Bahía de Juli, sector de Huaquina, comunidades de Olla y C'aje Huaylluni, 2006.

\section{Agradecimientos}

Este trabajo fue posible gracias al financiamiento del Ing. Pedro Grandarillas; asímismo agradececemos el apoyo en la revisión del manuscrito al Ms. Cs. Victor Quipuscoa, Bach. Edgardo Ortiz y Bach. Yeselia Cano por su apoyo en la determinación y sistematización de las Pteridophyta.

Agradecemos también al Blgo. Gilberto Carrasco, Sra. Carmen Beltrán, Sra. Sonia Gandarillas, Dr. Luis Arteta, Sr. Christian Arteta, Sr. Ray Calisaya por su apoyo logístico, a la Dra. Clara Figueroa, Decana de la Facultad de Ciencias de la Universidad Nacional Agraria La Molina, por su apoyo moral e impulsarnos a la realización del presente artículo, y a todas las personas que de una u otra manera hicieron posible la realización del presente estudio.

\section{Literatura Citada}

Brako L. \& Zarucchi J. 1993. Catálogo de Angiospermas y Gimnospermas del Perú. Missouri Botanical Garden. USA.

Cronquist A. 1981. An integrated system of classification of flowering plants. Columbia University Press. USA.

Holdridge L.P. 1978. Ecología basada en las zonas de vida. IICA, San José, Costa Rica.

INRENA. 1977. Listado de flora amenazada del Perú. D.S. 01710-77-AG. Lima - Perú.

INRENA. 2002. Plan Maestro de la Reserva Nacional del Titicaca. Puno - Perú.

León B. 1996. Propuesta de áreas importantes para la conservación de la diversidad de Pteridofitos en el Perú. En: Diversidad Biológica del Perú. Zonas prioritarias para su conservación. Pp. 44-47. INRENA - Perú.

León B. \& Young K. 1996. Distribution of Pteridophyte diversity and endemism in Perú. En: Pteridology in perspective. Pp. 77-91. USA.

Odum E. 1972. Ecología. $3^{\text {a }}$ Edición. Ed. Interamericana Mc Graw-Hill. Mexico, D. F.

Schjellerup I., Quipuscoa V., Espinoza C., Peña V. \&. Sorensen M.K 2005. Redescubriendo el valle de los Chilchos. Condiciones de vida en la Ceja de Selva, Perú. Ethnographic Monographs, No. 2. Trujillo - Perú.

Tryon R. \& Stolze R. 1989. Fieldiana Pterydophyta of Peru. Field Museum of Natural History. USA.

Valdivia J. 2005. Diagnóstico Situacional de la Contaminación en las Ciudades de Juli, Pomata y Yunguyo. Gobierno Regional Puno, Gerencia Regional de Recursos Naturales y Gestión del Medio Ambiente. Puno - Perú. 


\section{ANEXO 1}

Lista de especies de Magnoliophyta en la Bahía de Juli, sector de Huaquina, comunidades de Olla y C’aje - Huaylluni.

\begin{tabular}{|c|c|c|c|c|c|c|c|}
\hline FAMILIA & ESPECIE & 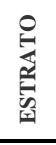 & 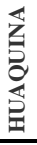 & 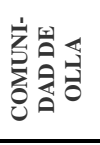 & 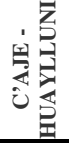 & \begin{tabular}{l}
0 \\
0 \\
\multirow{J}{J}{}
\end{tabular} & 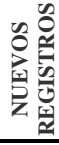 \\
\hline Alstroemeriaceae & Bomarea sp. & $\mathrm{H}$ & $x$ & & & & \\
\hline \multirow[t]{2}{*}{ Amaranthaceae } & Gomphrena meyeniana (Rusby) Süsseng. & $\mathrm{H}$ & $\mathrm{x}$ & & $\mathrm{x}$ & & \\
\hline & Gomphrena sp. & $\mathrm{H}$ & & & $\mathrm{x}$ & & \\
\hline \multirow[t]{5}{*}{ Apiaceae } & Bowlesia sodiroana Wolf, $\mathrm{H}$. & $\mathrm{H}$ & $\mathrm{x}$ & & & & $\mathrm{X}$ \\
\hline & Bowlesia tropaeolifolia Gillies \& Hook. & $\mathrm{H}$ & $\mathrm{x}$ & $\mathrm{x}$ & $\mathrm{x}$ & & \\
\hline & Ciclospermum laciniatum (DC.) Constante & $\mathrm{H}$ & $\mathrm{x}$ & & $\mathrm{x}$ & & $\mathrm{X}$ \\
\hline & Daucus montanus Humb. \& Bonpl. ex Sprengel & $\mathrm{H}$ & $\mathrm{x}$ & $\mathrm{x}$ & $\mathrm{x}$ & & $\mathrm{x}$ \\
\hline & Hydrocotile sp. & $\mathrm{H}$ & $\mathrm{x}$ & & & & \\
\hline \multirow[t]{40}{*}{ Asteraceae } & Acanthoxanthium spinosum (L.) Fourr. & $\mathrm{AT}$ & $\mathrm{x}$ & $\mathrm{x}$ & & & $\mathrm{x}$ \\
\hline & Achyroclyne alata (Kunth) DC. & AT & $\mathrm{x}$ & $\mathrm{x}$ & & & \\
\hline & Ageratina sternbergiana (DC.) King \& Robinson, H. & AT & $\mathrm{x}$ & $\mathrm{x}$ & $\mathrm{x}$ & & \\
\hline & Ambrosia arborescens Miller & AT & $\mathrm{x}$ & $\mathrm{x}$ & & & $\mathrm{x}$ \\
\hline & Baccharis tricuneata (L.f.) Persoon & AT & $\mathrm{x}$ & $\mathrm{x}$ & $\mathrm{x}$ & & \\
\hline & Bidens pilosa L. & $\mathrm{H}$ & & $\mathrm{x}$ & & & \\
\hline & Bidens andicola Kunth & $\mathrm{H}$ & $\mathrm{x}$ & $\mathrm{x}$ & $\mathrm{x}$ & & \\
\hline & Chersodoma jodopappa (Schultz-Bip.) Cabrera & $\mathrm{H}$ & $\mathrm{x}$ & & $\mathrm{x}$ & & $\mathrm{x}$ \\
\hline & Conyza artemisiifolia Meyen \& Walp. & $\mathrm{H}$ & $\mathrm{x}$ & $\mathrm{x}$ & $\mathrm{x}$ & & \\
\hline & Conyza sp. & $\mathrm{H}$ & $\mathrm{x}$ & & $\mathrm{x}$ & & \\
\hline & Cosmos peucedanifolius Wedd. & $\mathrm{H}$ & & & $\mathrm{x}$ & & $\mathrm{x}$ \\
\hline & Cotula australis (Sieber ex Sprengel) Hook.f. & $\mathrm{H}$ & $\mathrm{x}$ & $\mathrm{x}$ & & & $\mathrm{x}$ \\
\hline & Cotula coronopifolia L. & $\mathrm{H}$ & $\mathrm{x}$ & & & & \\
\hline & Galinsoga parviflora Cav. & $\mathrm{H}$ & $\mathrm{x}$ & & $\mathrm{x}$ & & $\mathrm{x}$ \\
\hline & Galinsoga sp. & $\mathrm{H}$ & & $\mathrm{x}$ & & & \\
\hline & Gamochaeta americana (Millar) Wedd. & $\mathrm{H}$ & $\mathrm{x}$ & $\mathrm{x}$ & $\mathrm{x}$ & & \\
\hline & Gamochaeta sp. & $\mathrm{H}$ & $\mathrm{x}$ & & $\mathrm{x}$ & & \\
\hline & Grindelia sp. & AT & & & $\mathrm{x}$ & & \\
\hline & Hieracium sp. & $\mathrm{H}$ & $\mathrm{x}$ & $\mathrm{x}$ & $\mathrm{x}$ & & \\
\hline & Hypochaeris meyeniana (Walp.) Grises. & $\mathrm{H}$ & & $\mathrm{x}$ & & & $\mathrm{x}$ \\
\hline & Hypochaeris sp.1 & $\mathrm{H}$ & $\mathrm{x}$ & & & & \\
\hline & Hypochaeris sp.2 & $\mathrm{H}$ & $\mathrm{x}$ & & $\mathrm{x}$ & & \\
\hline & Mutisia cochabambensis Hieron. & SV & $\mathrm{x}$ & & & & $\mathrm{x}$ \\
\hline & $\begin{array}{l}\text { Ophryosporus heptanthus (Schultz-Bip. ex Wedd.) } \\
\text { King \& Robinson, H. }\end{array}$ & AT & $\mathrm{x}$ & & $\mathrm{x}$ & & \\
\hline & Perezia multiflora (Humb. \& Bonpl.) Less. & $\mathrm{H}$ & $\mathrm{x}$ & $\mathrm{x}$ & $\mathrm{x}$ & & \\
\hline & Pseudognaphalium lacteum Meyen \&Walpers & $\mathrm{H}$ & & & $\mathrm{x}$ & & \\
\hline & Pseudognaphalium sp.1 & $\mathrm{H}$ & $\mathrm{x}$ & $\mathrm{x}$ & $\mathrm{x}$ & & \\
\hline & Pseudognaphalium sp.2 & $\mathrm{H}$ & $\mathrm{x}$ & & & & \\
\hline & Pseudognaphalium sp.3 & $\mathrm{H}$ & & $\mathrm{x}$ & & & \\
\hline & Senecio vulgaris L. & $\mathrm{AT}$ & $\mathrm{x}$ & $\mathrm{x}$ & $\mathrm{x}$ & & $\mathrm{x}$ \\
\hline & Silybum marianum (L.) Gaertner & $\mathrm{H}$ & & $\mathrm{x}$ & & & \\
\hline & Sonchus asper (L.) Hill & $\mathrm{H}$ & $\mathrm{x}$ & $\mathrm{x}$ & & & \\
\hline & Sonchus oleraceus L. & $\mathrm{H}$ & $\mathrm{x}$ & $\mathrm{x}$ & $\mathrm{x}$ & & \\
\hline & Stevia sp. & $\mathrm{H}$ & $\mathrm{x}$ & $\mathrm{x}$ & & & \\
\hline & Tanacetum parthenium (L.) Schultz-Bip. & AT & $\mathrm{x}$ & & & & \\
\hline & Tagetes filifolia Lagasca & $\mathrm{H}$ & $\mathrm{x}$ & $\mathrm{x}$ & $\mathrm{x}$ & & \\
\hline & Tagetes multiflora Kunth & $\mathrm{H}$ & $\mathrm{x}$ & $\mathrm{x}$ & & & $\mathrm{x}$ \\
\hline & Taraxacum officinale Wigg. & $\mathrm{H}$ & $\mathrm{x}$ & $\mathrm{x}$ & $\mathrm{x}$ & & $\mathrm{x}$ \\
\hline & Viguiera lanceolata Britton & $\mathrm{SF}$ & $\mathrm{x}$ & $\mathrm{x}$ & $\mathrm{x}$ & & $\mathrm{x}$ \\
\hline & Viguiera sp. & $\mathrm{SF}$ & $\mathrm{x}$ & & $\mathrm{x}$ & & \\
\hline Boraginaceae & Heliotropium sp. & $\mathrm{H}$ & $\mathrm{x}$ & & & & \\
\hline \multirow[t]{7}{*}{ Brassicaceae } & Brassica rapa subsp. campestris (L.) Clapham & $\mathrm{H}$ & $\mathrm{x}$ & & & & $\mathrm{x}$ \\
\hline & Capsella bursa-pastoris (L.) Medicus & $\mathrm{H}$ & $\mathrm{x}$ & $\mathrm{x}$ & & & \\
\hline & $\begin{array}{l}\text { Descurainia myriophylla (Willd. ex DC.) } \\
\text { Fries, R.(E.) }\end{array}$ & $\mathrm{H}$ & $\mathrm{x}$ & & & & \\
\hline & Lepidium bipinnatifidum Desv. & $\mathrm{H}$ & $\mathrm{x}$ & $\mathrm{x}$ & & & \\
\hline & Lepidium sp. & $\mathrm{H}$ & & & $\mathrm{x}$ & & \\
\hline & Sisymbrium gracile Wedd. & $\mathrm{H}$ & $\mathrm{x}$ & & & & $\mathrm{x}$ \\
\hline & Sysimbrium sp. & $\mathrm{H}$ & & & $\mathrm{x}$ & & \\
\hline Bromeliacae & Tyllandsia capillaris R.\&P. & $\mathrm{H}$ & $\mathrm{x}$ & & & & \\
\hline
\end{tabular}


PLANTAS VASCULARES DE LA BAHÍA DE JULI, LAGO TITICACA, PUNO - PERÚ 2006

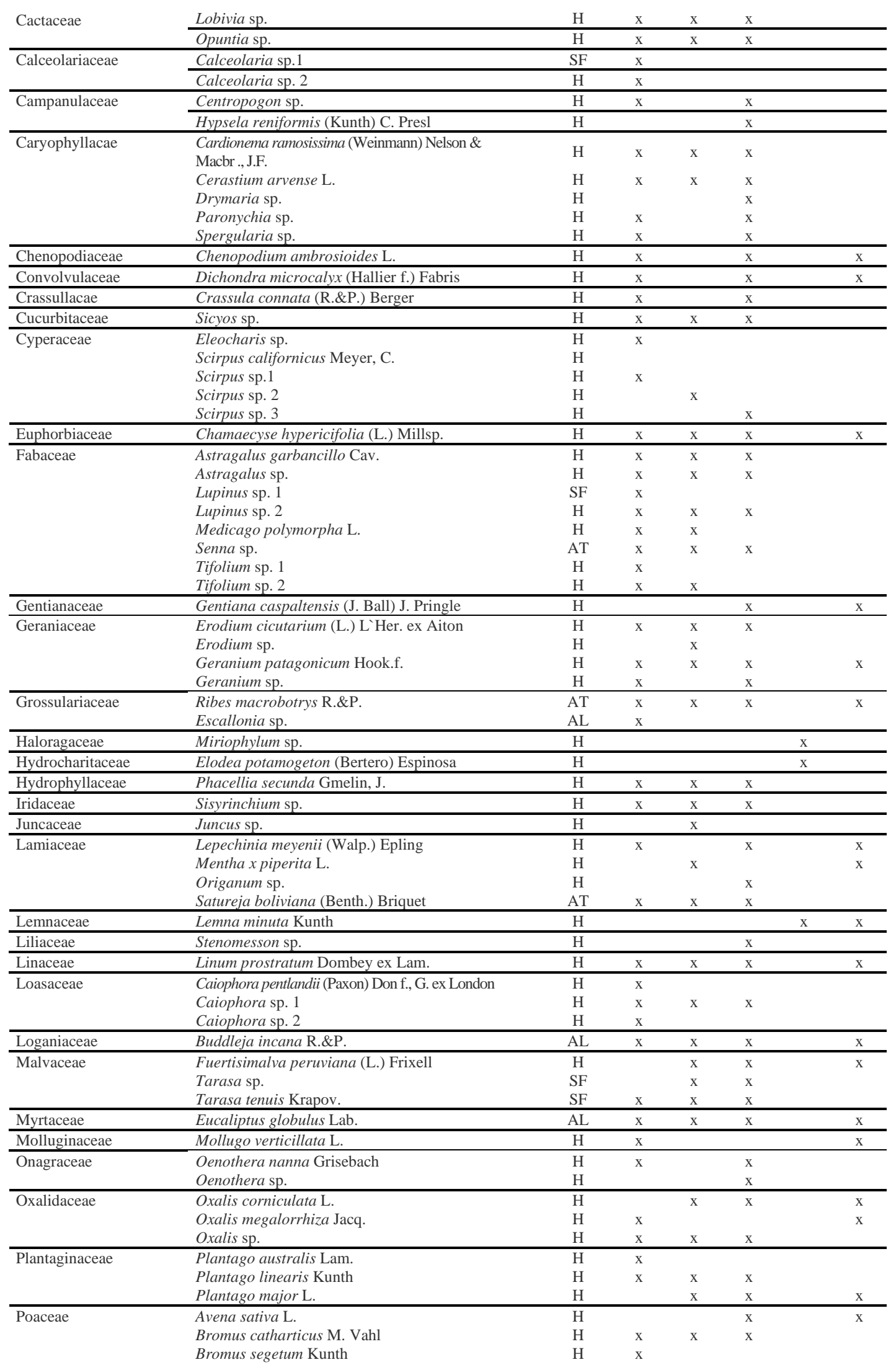




\begin{tabular}{|c|c|c|c|c|c|c|c|}
\hline & Calamagrostis curvula (Wedd.) Pilger & $\mathrm{H}$ & $\mathrm{x}$ & & & & \\
\hline & Chondrosum simplex (Lagasca) Kunth & $\mathrm{H}$ & $\mathrm{x}$ & $\mathrm{x}$ & $\mathrm{x}$ & & \\
\hline & Cortaderia jubata (Lemaire) Stanpfinhook f.. & $\mathrm{H}$ & $\mathrm{x}$ & & $\mathrm{x}$ & & $\mathrm{x}$ \\
\hline & Eragrostis nigricans (Kunth) Steudel & $\mathrm{H}$ & $\mathrm{x}$ & $\mathrm{x}$ & & & $\mathrm{x}$ \\
\hline & Eragrostis soratensis Jedwadnik & $\mathrm{H}$ & $\mathrm{x}$ & $\mathrm{x}$ & & & \\
\hline & Eragrostis sp. & $\mathrm{H}$ & $\mathrm{x}$ & $\mathrm{x}$ & $\mathrm{x}$ & & \\
\hline & Festuca ortophylla Pilger & $\mathrm{H}$ & & $\mathrm{x}$ & $\mathrm{x}$ & & \\
\hline & Hordeum muticum Presl., J. & $\mathrm{H}$ & & & $\mathrm{x}$ & & \\
\hline & Hordeum vulgare L. & $\mathrm{H}$ & $\mathrm{x}$ & $\mathrm{x}$ & $\mathrm{x}$ & & $\mathrm{x}$ \\
\hline & Paspalidium sp. & $\mathrm{H}$ & $\mathrm{x}$ & & & & \\
\hline & Poa апnиа $\mathrm{L}$. & $\mathrm{H}$ & $\mathrm{x}$ & $\mathrm{x}$ & $\mathrm{x}$ & & \\
\hline & Poa sp. & $\mathrm{H}$ & & & $\mathrm{x}$ & & \\
\hline & Polypogon interruptus Kunth & $\mathrm{H}$ & $\mathrm{x}$ & & & & \\
\hline & Stipa ichu (R. \& P.) Kunth & $\mathrm{H}$ & $\mathrm{x}$ & $\mathrm{x}$ & & & \\
\hline & Stipa sp. & $\mathrm{H}$ & $\mathrm{x}$ & $\mathrm{x}$ & & & \\
\hline \multirow[t]{3}{*}{ Polemoniaceae } & Gilia laciniata R.\&P. & $\mathrm{H}$ & $\mathrm{x}$ & $\mathrm{x}$ & & & \\
\hline & Phlox gracilis (Douglas ex Hook.) Greene, E. & $\mathrm{H}$ & $\mathrm{x}$ & & & & $\mathrm{x}$ \\
\hline & Phlox sp. & $\mathrm{H}$ & & & $\mathrm{x}$ & & \\
\hline \multirow[t]{3}{*}{ Polygonaceae } & Muehlenbeckia volcanica (Benth.) Ende. & $\mathrm{SF}$ & $\mathrm{x}$ & $\mathrm{x}$ & $\mathrm{x}$ & & \\
\hline & Rumex crispus L. & $\mathrm{H}$ & $\mathrm{x}$ & & $\mathrm{x}$ & & $\mathrm{x}$ \\
\hline & Rumex obtusifolius L. & $\mathrm{H}$ & & $\mathrm{x}$ & & & $\mathrm{x}$ \\
\hline \multirow[t]{2}{*}{ Portulacaceae } & Portulaca sp. & $\mathrm{H}$ & $\mathrm{x}$ & & & & \\
\hline & $\begin{array}{l}\text { Portulaca oleracea subsp. tuberculata Danin \& H. G. } \\
\text { Baker }\end{array}$ & $\mathrm{H}$ & $\mathrm{x}$ & & & & $\mathrm{x}$ \\
\hline Rhamnaceae & Colletia spinosissima J. Gmelin & $\mathrm{H}$ & $\mathrm{x}$ & $\mathrm{x}$ & $\mathrm{x}$ & & \\
\hline \multirow[t]{4}{*}{ Rosaceae } & Alchemilla sp. & $\mathrm{H}$ & & $\mathrm{x}$ & $\mathrm{x}$ & & \\
\hline & Polylepis incana Kunth & $\mathrm{AL}$ & $\mathrm{x}$ & & & & $\mathrm{x}$ \\
\hline & Rosa sp. & AT & $\mathrm{x}$ & $\mathrm{x}$ & & & \\
\hline & Tetraglochin cristatum (Britt.) Rothm. & $\mathrm{AT}$ & $\mathrm{x}$ & $\mathrm{x}$ & $\mathrm{x}$ & & \\
\hline Rubiaceae & Galium sp. & $\mathrm{H}$ & & $\mathrm{x}$ & $\mathrm{x}$ & & \\
\hline \multirow[t]{3}{*}{ Scrophulariaceae } & Bartsia diffusa Benth. & $\overline{\mathrm{H}}$ & $\mathrm{x}$ & $\bar{x}$ & $\bar{x}$ & & \\
\hline & Castilleja sp. & $\mathrm{H}$ & & $\mathrm{x}$ & & & \\
\hline & Mimulus glabratus Kunth & $\mathrm{H}$ & & & $\mathrm{x}$ & & $\mathrm{x}$ \\
\hline \multirow[t]{6}{*}{ Solanaceae } & Nicotiana paniculata L. & $\mathrm{SF}$ & $\mathrm{x}$ & & $\mathrm{x}$ & & $\bar{x}$ \\
\hline & Nicotiana sp. & $\mathrm{SF}$ & & $\mathrm{x}$ & $\mathrm{x}$ & & \\
\hline & Salpichroa sp. & SF & $\mathrm{x}$ & $\mathrm{x}$ & $\mathrm{x}$ & & \\
\hline & Solanum nitidum R.\&P. & $\mathrm{AT}$ & $\mathrm{x}$ & $\mathrm{x}$ & $\mathrm{x}$ & & \\
\hline & Solanum sp.1 & $\mathrm{H}$ & $\mathrm{x}$ & $\mathrm{x}$ & & & \\
\hline & Solanum sp. 2 & $\mathrm{H}$ & $\mathrm{x}$ & $\mathrm{x}$ & $\mathrm{x}$ & & \\
\hline Urticaceae & Urtica leptophylla Kunth & $\mathrm{H}$ & $\mathrm{x}$ & $\mathrm{x}$ & $\mathrm{x}$ & & \\
\hline \multirow[t]{2}{*}{ Verbenaceae } & Glandularia sp. & $\mathrm{H}$ & $\mathrm{x}$ & $\mathrm{x}$ & $\mathrm{x}$ & & \\
\hline & Verbena hispida R. \& P. & $\mathrm{H}$ & $\mathrm{x}$ & $\mathrm{x}$ & $\mathrm{x}$ & & $\mathrm{x}$ \\
\hline Violaceae & Viola sp. & $\mathrm{H}$ & & $\mathrm{x}$ & & $\mathrm{x}$ & \\
\hline Zannichelliaceae & Zannichellia sp. & $\mathrm{H}$ & & $\mathrm{x}$ & & $\mathrm{x}$ & \\
\hline
\end{tabular}

AT= Arbusto, $\mathrm{AL}=$ Árbol, $\mathrm{H}=$ Hierba, $\mathrm{SF}=$ Sufrútice, $\mathrm{SV}=$ Sufrútice Voluble

ANEXO 2

Listado de especies de Pteridophyta presentes en el Sector de Huaquina, Comunidades de Olla y C'aje Huaylluni, en la Bahía de Juli, Lago Titicaca, Departamento de Puno, 2006. H= Hierba

\begin{tabular}{|c|c|c|c|c|c|c|c|}
\hline FAMILIA & ESPECIE & 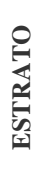 & 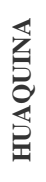 & 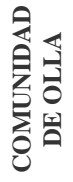 & 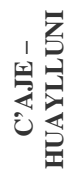 & 足 & 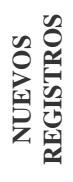 \\
\hline Adiantaceae & Adianthum sp. & $\mathrm{H}$ & & $\mathrm{x}$ & $\mathrm{x}$ & & \\
\hline Aspleniaceae & Asplenium sp. & $\mathrm{H}$ & $\mathrm{x}$ & $\mathrm{x}$ & $\mathrm{x}$ & & \\
\hline \multirow{4}{*}{ Dryopteridaceae } & Cystopteris fragilis (L.) Bernh. & $\mathrm{H}$ & $\mathrm{x}$ & $\mathrm{x}$ & & & \\
\hline & Cystopteris sp. & $\mathrm{H}$ & $\mathrm{x}$ & & & & \\
\hline & Polystichum sp. & $\mathrm{H}$ & $\mathrm{x}$ & $\mathrm{x}$ & & & \\
\hline & Woodsia sp. & $\mathrm{H}$ & $\mathrm{x}$ & & & $\mathrm{x}$ & \\
\hline Equisetaceae & Equisetum bogotense Kunth. & $\mathrm{H}$ & $\mathrm{x}$ & & & $\mathrm{x}$ & \\
\hline \multirow{4}{*}{ Pteridaceae } & Pellaea ternifolia (Cav.) Link & $\mathrm{H}$ & & $\mathrm{x}$ & $\mathrm{x}$ & & \\
\hline & Argyrochosma nivea (Poiret) Windham & $\mathrm{H}$ & & $\mathrm{x}$ & & & \\
\hline & Cheylanthes pruinata Kaulf. & $\mathrm{H}$ & $\mathrm{x}$ & & $\mathrm{x}$ & & \\
\hline & Eriosorus sp. & $\mathrm{H}$ & $\mathrm{x}$ & $\mathrm{x}$ & $\mathrm{x}$ & & \\
\hline Salviniaceae & Azolla filiculoides Lam. & $\mathrm{H}$ & & $\mathrm{x}$ & & $\mathrm{x}$ & $\mathrm{x}$ \\
\hline
\end{tabular}




\section{ANEXO 3}

Listado de especies de Pinophyta presentes en el Sector de Huaquina, Comunidades de Olla y C’aje - Huaylluni, en la Bahía de Juli, Lago Titicaca, Departamento de Puno, 2006

\begin{tabular}{|c|c|c|c|c|c|c|c|}
\hline FAMILIA & ESPECIE & 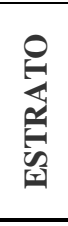 & 晃 & 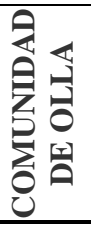 & 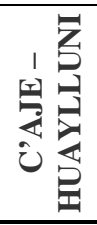 & 帠 & 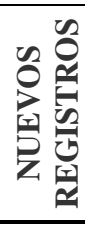 \\
\hline Pinaceae & Pinus radiata $\mathrm{L}$. & $\mathrm{AL}$ & $\mathrm{x}$ & $\mathrm{x}$ & $\mathrm{x}$ & & \\
\hline Cupressaceae & Cupresus sempervirens L. & $\mathrm{AL}$ & $\mathrm{x}$ & $\mathrm{x}$ & $\mathrm{x}$ & & \\
\hline Ephedraceae & $\begin{array}{l}\text { Ephedra americana Humb.\& } \\
\text { Bonpl. ex Willd. }\end{array}$ & $\mathrm{SF}$ & $\mathrm{x}$ & $\mathrm{X}$ & & & \\
\hline
\end{tabular}

$\mathrm{AL}=$ Árbol, $\mathrm{SF}=$ Sufrútice.

${ }^{1}$ Herbarium Areqvipense (HUSA) - Universidad Nacional de San Agustín de Arequipa. Av. Alcides Carrión s/n. Arequipa;

${ }^{2}$ Herbarium Areqvipense (HUSA) - Universidad Nacional de San Agustín de Arequipa. Av. Alcides Carrión s/n. Arequipa; massiel_cm@hotmail.com

${ }^{3}$ Herbarium Areqvipense (HUSA) - Universidad Nacional de San Agustín de Arequipa. Av. Alcides Carrión s/n. Arequipa; c.davalos.m@gmail.com

${ }^{4}$ Herbarium Areqvipense (HUSA) - Universidad Nacional de San Agustín de Arequipa. Av. Alcides Carrión s/n. Arequipa; amy_cdr@hotmail.com

${ }^{5}$ Herbarium Areqvipense (HUSA) - Universidad Nacional de San Agustín de Arequipa. Av. Alcides Carrión s/n. Arequipa; fsincac@gmail.com

${ }^{6}$ Herbarium Areqvipense (HUSA) - Universidad Nacional de San Agustín de Arequipa. Av. Alcides Carrión s/n. Arequipa; liz_ha1@hotmail.com

${ }^{7}$ Facultad de Ciencias de la Universidad Nacional Agraria La Molina; bojorquez.javier@gmail.com 